\title{
Retrospective study on prevalence of recurrent inguinal hernia: a large-scale multi-institutional study
}

\author{
Chul Seung Lee', Ji Hoon Kim², Byung Jo Choi ${ }^{3}$, Jae Im Lee ${ }^{4}$, Sang Chul Lee ${ }^{3}$, Yoon Suk Lee', \\ Seong Taek $\mathrm{Oh}^{4}$, Hyung Jin Kim ${ }^{5}$ \\ ${ }^{1}$ Department of Surgery, Seoul St. Mary's Hospital, College of Medicine, The Catholic University of Korea, Seoul, Korea \\ ${ }^{2}$ Department of Surgery, Incheon St. Mary's Hospital, College of Medicine, The Catholic University of Korea, Incheon, Korea \\ ${ }^{3}$ Department of Surgery, Daejeon St. Mary's Hospital, College of Medicine, The Catholic University of Korea, Daejeon, Korea \\ ${ }^{4}$ Department of Surgery, Uijeongbu St. Mary's Hospital, College of Medicine, The Catholic University of Korea, Uijeongbu, Korea \\ ${ }^{5}$ Department of Surgery, Eunpyeong St. Mary's Hospital, College of Medicine, The Catholic University of Korea, Seoul, Korea
}

\begin{abstract}
Purpose: We conducted a multi-institutional analysis to establish the epidemiological characteristics of recurrent inguinal hernia following hernia repair in patients across 4 institutions in Korea.

Methods: The retrospectively reviewed data included patient characteristics, hernia location, year of primary operation, type of hernia, timing of recurrence, primary operation type, and whether a mesh was used.

Results: Among 4,604 patients who underwent hernia repair surgery, 255 patients (5.5\%; 13 females and 242 males; mean age, 63 years) were found to have recurrent hernia from January 2010 to April 2017. Recurrent indirect inguinal and direct hernias were observed in $47.1 \%$ and $49.4 \%$ of the patients, respectively. The recurrence of hernias within 1 year of surgery was the highest at $17.25 \%$. Early and late recurrences was observed in $23.5 \%$ and $66.5 \%$ of the patients, respectively. Among the patients, $81.6 \%$ underwent open hernia repair at the time of initial surgery.

Conclusion: Recurrence of hernia is most common in the first year after the initial surgery, and $23.5 \%$ of recurrent inguinal hernia was developed within 2 years. Patients underwent surgery after an average of 116 months Imedian value, 64 months) following the first operation. In patients with recurrent hernia, direct hernia was seen more frequent than indirect hernia whereas indirect hernia occurred more in patients with primary hernia.

[Ann Surg Treat Res 2020;98(1):51-55]
\end{abstract}

Key Words: Herniorrhaphy, Inguinal hernia, Recurrence

\section{INTRODUCTION}

The recurrence rate of inguinal hernia following primary hernia repair ranges from $0.5 \%$ to $15 \%$ depending upon the hernia site, the type of repair, and the clinical circumstances [1-5]. Although the recurrence rate of inguinal hernia may be declining due to the more frequent use of mesh in primary hernia repairs [6], recurrence still occurs due to various factors and it clearly remains a major health problem.

Early recurrence (within 2 years) is generally related to technical factors [6]. The main technical factors commonly associated with recurrent inguinal hernia are related to either a tissue repair $[4,7,8]$ or inadequate mesh size or inadequate mesh fixation. Tissue repair, which is less likely to produce a tension-
Received July 23, 2019, Revised November 2, 2019,

Accepted November 15, 2019

Corresponding Author: Hyung Jin Kim

Department of Surgery, Eunpyeong St. Mary's Hospital, College of Medicine, The Catholic University of Korea, 1021 Tongil-ro, Eunpyeong-gu, Seoul 03312, Korea

Tel: +82-2-2030-4389, Fax: +82-2-2030-4647

E-mail: hj@catholic.ac.kr

ORCID: https://orcid.org/0000-0002-6315-714X

\section{Copyright (c) 2020, the Korean Surgical Society}

(c) Annals of Surgical Treatment and Research is an Open Access Journal. All articles are distributed under the terms of the Creative Commons Attribution NonCommercial License (http://creativecommons.org/licenses/by-nc/4.0/) which permits unrestricted non-commercial use, distribution, and reproduction in any medium, provided the original work is properly cited. 
free repair, is an important cause of failed hernia repair. No significant differences have been identified in the incidence of recurrent hernia following primary hernia repair using a mesh, regardless of operative approach such as open or laparoscopic hernia repair $[2,4,5,9,10]$.

Late recurrences (after 2 years) are usually related to patient-related factors. Patient factors that increase the risk for recurrent inguinal hernia are generally those that disrupt or weaken the tissues, contribute to poor wound healing, or increase the risk for postoperative infection. There have been many developments in the surgical procedure for inguinal hernia; presently, the so-called 'tension-free repair' is the procedure of choice [11] owing to the low recurrence rate of hernias associated with it. However, there have been few reports on recurrence in Asia after laparoscopic and open tension-free methods have become common. Although laparoscopic and open surgical techniques are evolving in form and number, there is a lack of epidemiologic data for recurrent hernia in Asia, including Korea. Therefore, we conducted this large-scale study in 4 hospitals where mesh surgery was being actively performed.

This study aimed to investigate the epidemiological characteristics of recurrent inguinal hernia in patients across these 4 institutions.

\section{METHODS}

We performed a retrospective review of 255 patients who underwent repair of recurrent inguinal hernia in the Department of Surgery at St. Vincent's Hospital, Uijeongbu St. Mary's Hospital, Daejeon St. Mary's Hospital, and Incheon St. Mary's Hospital from January 2010 to April 2017. The diagnosis of recurrent inguinal hernia was established by clinical data (history and physical examination), and ultrasonographic or CT findings. Patient inclusion criteria were age $>12$ years, admission in the Surgery Department, American Society of

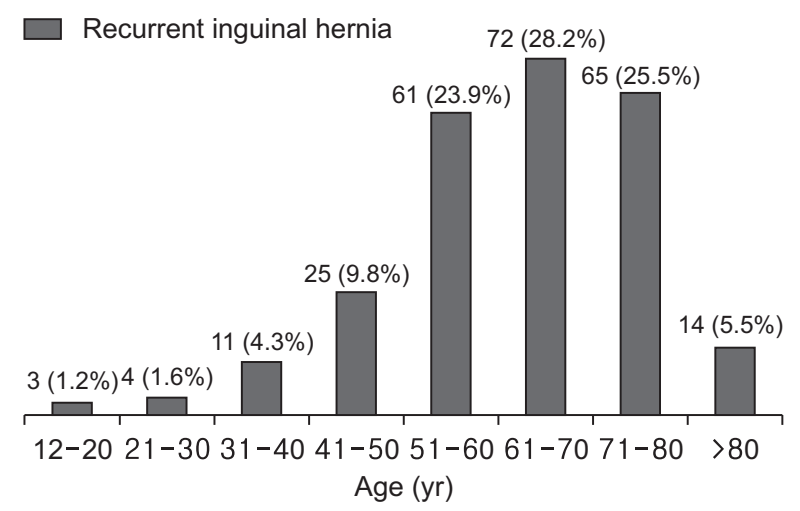

Fig. 1. Prevalence of recurrent inguinal hernias in patients of different age groups.
Anesthesiologists physical status classification I or II, and cases of inguinal hernia only (direct:indirect:mixed). Exclusion criteria were age $<12$ years, American Society of Anesthesiologists physical status classification III or worse, and emergency repair for outlet obstruction or strangulation. Patients returned to the outpatient department on the seventh day after discharge for an evaluation of their general condition. This study was approved by the Catholic Medical Center Research Ethics Board (IRB No. XC19REDI0054). Informed consent from patients to be included in this study was omitted according to the policy of our IRB. Statistical analysis was performed using IBM SPSS Statistics ver. 24.0 (IBM Co., Armonk, NY, USA). Differences between groups were evaluated using Student t-test and the chi-square test for continuous and categorical variables, respectively. P-values of $<0.05$ were considered as statistically significant.

\section{RESULTS}

Among 4,604 patients who underwent hernia surgery, 255 patients (5.54\%, 13 females and 242 males) were found to have recurrent hernia. Of them, 43 patients (16.9\%) were under 50 years of age. Recurrent hernias were found predominantly between the ages of 61 to 70 , comprising $28.2 \%$ of total recurrent hernia cases as shown in Fig. 1. Right-sided recurrent hernias $(150,58.8 \%)$ seemed to be more prevalent than leftsided ones $(87,34.1 \%)$. Bilateral recurrent inguinal hernias were reported in 9 patients (3.5\%) (Table 1).

Indirect recurrent inguinal hernia occurred in $47.1 \%$ of the patients and direct hernia in $49.4 \%$. Average time to recurrence was 9 years and 8 months. The incidence of early $(<2$ years after primary hernia) recurrence was $23.5 \%$. The recurrence of hernias within 1 year of surgery was the highest at $17.3 \%$, followed by those presenting 1 to 2 years after (7.5\%), 2 to 3

Table 1. Baseline characteristics of recurrent inguinal hernia

\begin{tabular}{lc}
\hline \multicolumn{1}{c}{ Characteristic } & Value \\
\hline Age $(\mathrm{yr})$ & $62.62 \pm 13.65$ \\
Sex & $242(94.9)$ \\
Male & $13(5.1)$ \\
Female & \\
Site & $150(58.8)$ \\
Right & $87(34.1)$ \\
Left & $9(3.5)$ \\
Bilateral & $9(3.5)$ \\
Unknown & \\
Type of recurrence & $61(23.5)$ \\
Early $(<2$ years after primary hernia) & $173(66.5)$ \\
Late $(\geq 2$ years after primary hernia) & $21(8.1)$ \\
Unknown &
\end{tabular}

Values are presented as mean \pm standard deviation or number (\%). 
years after (7.1\%), and 3 to 4 years after (7.1\%) the operation. After 4 years of the primary hernia operation, the recurrence rate decreased to less than $7 \%$ (Fig. 2).

Of the patients presenting with recurrent hernias, $81.6 \%$ had

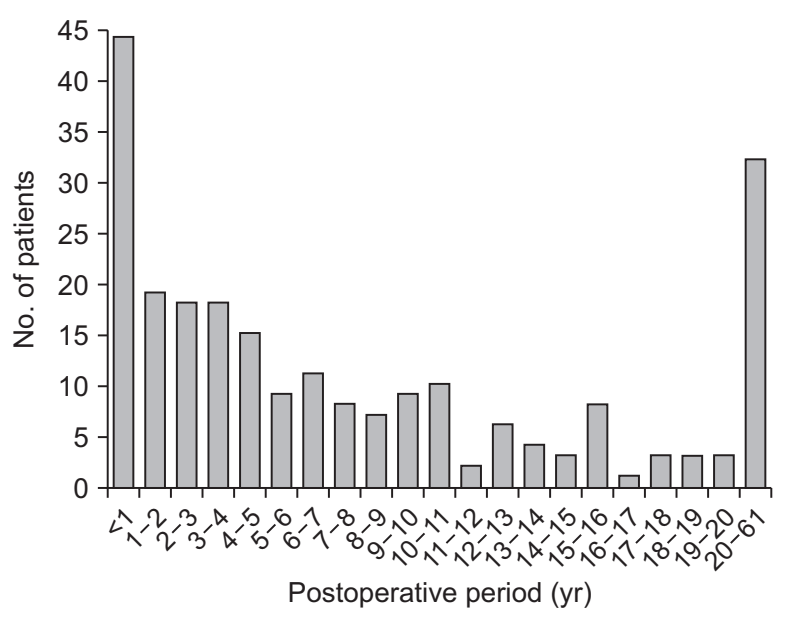

Fig. 2. Timing of hernia recurrence. undergone open methods at the time of initial surgery and $18.4 \%$ had undergone laparoscopic surgery. The initial surgery was performed at other hospitals for $61.2 \%$ of patients with

Table 2. Operation method and hospital of primary hernia repair

\begin{tabular}{lr}
\hline Primary hernia repair & No. $(\%)$ \\
\hline Operation method & \\
Open & \\
Laparoscopic & $47(18.4)$ \\
Total & $255(100)$ \\
Hospital & \\
Participating institutes ${ }^{\mathrm{a})}$ & $99(38.8)$ \\
Outside & $156(61.2)$ \\
Mesh & $100(39.2)$ \\
Yes & $93(36.5)$ \\
No & $62(24.3)$ \\
Unknown & \\
\hline
\end{tabular}

${ }^{a}$ St. Vincent's Hospital, Uijeongbu St. Mary's Hospital, Daejeon St. Mary's Hospital, and Incheon St. Mary's Hospital.

Table 3. Primary operation with and without mesh

\begin{tabular}{lccc}
\hline \multicolumn{1}{c}{ Variable } & Mesh repair & Tissue repair & P-value \\
\hline Male sex & $94(94.0)$ & $75(96.1)$ & 0.515 \\
Age $>60 \mathrm{yr}$ & $64(64.0)$ & $44(56.4)$ & 0.354 \\
Primary hernia type & & & \\
$\quad$ Direct:indirect:femoral & $50: 46: 1(51.0: 46.9: 1.0)$ & $40: 36: 0(52.6: 47.3: 0)$ & 0.844 \\
Primary hernia location & $57: 35: 8(57.0: 35.0: 8.0)$ & $45: 27: 6(57.7: 34.6: 7.7)$ & 0.995 \\
$\quad$ Right:left:both & $59: 36: 4(59.6: 36.4: 4.0)$ & $46: 28: 2(60.5: 36.8: 2.6)$ & 0.879 \\
Recurrent hernia location & $44.2 \pm 49.3$ & $184.8 \pm 171.7$ & $<0.001$ \\
$\quad$ Right:left:both & & &
\end{tabular}

Values are presented as number $(\%)$ or mean \pm standard deviation.

Table 4. Comparison of early and late recurrence of hernia

Variable

No. of patients
Male sex
Age $>60$ yr
Primary hernia type
Direct:indirect:femoral
Primary hernia location
Right:left:both
Recurrent hernia location
Right:left:both
Primary repair
Mesh repair:tissue repair

\section{Early $(<2$ years after primary hernia)}

Late ( $\geq 2$ years after primary hernia)
P-value

$\begin{array}{lrl}61(23.5) & 173(66.5) & \\ 57(93.4) & 166(96.0) & 0.426 \\ 35(57.3) & 69(60.1) & 0.708\end{array}$

22:34:1 (37.9:58.6:1.7)

96:75:0 (56.1:43.9:0)

0.068

$31: 25: 5(50.8: 41.0: 8.2)$

104:54:15 (60.1:31.2:8.7)

0.375

$105: 57: 5^{\text {a) }}(62.9: 34.1: 3.0)$

0.560

$47: 9(83.9: 1.6)$

$53: 69(43.3: 56.6)$

$<0.001$

Values are presented as number (\%).

a) $\mathrm{n}=167$. 
Table 5. Comparison of primary surgery in participating institutes and in the other hospitals

\begin{tabular}{lccc}
\hline \multicolumn{1}{c}{ Variable } & $\begin{array}{c}\text { Primary surgery in } \\
\text { participating institutes }\end{array}$ & Primary surgery in other hospitals & P-value \\
\hline $\begin{array}{l}\text { Male sex } \\
\text { Age }>60 \text { yr }\end{array}$ & $125(94.7)$ & $98(96.1)$ & 0.621 \\
$\quad \begin{array}{l}\text { Primary hernia type } \\
\quad \text { Direct:indirect:femoral } \\
\text { Primary hernia location } \\
\quad \text { Right:left:both }\end{array}$ & $55(41.7)$ & $77(58.3)$ & 0.789 \\
$\quad \begin{array}{l}\text { Recurrent hernia location } \\
\quad \text { Right:left:both }\end{array}$ & $52: 46: 0(53.1: 46.9: 0)$ & $66: 63: 1(50.8: 48.5: 0.8)$ & 0.803 \\
$\quad \begin{array}{l}\text { Primary repair } \\
\quad \text { Mesh repair/tissue repair }\end{array}$ & $59: 34: 9(57.8: 33.3: 8.8)$ & $76: 45: 11(57.6: 34.1: 8.3)$ & 0.987 \\
$\quad$ Timing of hernia recurrence $(\mathrm{mo})$ & $74: 35: 3(62.0: 35.0: 3.0)$ & $76: 47: 4(59.8: 37.0: 3.1)$ & 0.947 \\
\hline
\end{tabular}

Values are presented as number $(\%)$ or mean \pm standard deviation.

Table 6. Operation methods for primary hernia and recurrent hernia

\begin{tabular}{lc}
\hline Operation methods for primary hernia and recurrent hernia & No. of cases (\%) \\
Open surgery for primary and recurrent hernia & $47(28.0)$ \\
Open surgery for primary hernia and laparoscopic surgery for recurrent hernia & $75(44.6)$ \\
Laparoscopic surgery for primary hernia and open surgery for recurrent hernia & $40(23.8)$ \\
Laparoscopic surgery for primary and recurrent hernia & $6(3.6)$ \\
\hline
\end{tabular}

recurrent hernia, while $38.8 \%$ had undergone initial surgery at our institutions. In the primary repair, 39.2\% of patients had undergone mesh repair and $36.5 \%$ of patients had undergone tissue repair (Table 2). Early recurrence rate was higher in the mesh repair group than in the tissue repair group $(\mathrm{P}<0.001)$ (Tables 3 and 4). The frequency of mesh repair was higher in participating institutes than in the other hospitals, and the time to recurrence was shorter in participating institutes group than others (Table 5). Open surgery was most commonly performed for primary hernia while laparoscopic surgery was commonly performed for recurrent hernia (Table 6).

\section{DISCUSSION}

According to nationwide data on inguinal hernia surgery from 2007 to 2015, there was a dramatic increase in laparoscopic inguinal hernia repair procedures from less than 3\% in 2007 to about 30\% in 2015 [12]. Every year about 35,000 inguinal hernia repairs are performed in Korea and the incidence peak was found in adult patients aged 50 to 70 years [12]. Similarly, recurrent hernias were found most often between the ages of 61 and 70 years.

Nationwide data from 2007 to 2015 showed that, in Korea, every year approximately $2 \%-3 \%$ of repair operations are performed for recurrent hernia [12]. From 2007 to 2015, a total of 311,441 operations for inguinal hernia were performed in Korea, which comprised of $87 \%$ in male patients and $13 \%$ in female patients [12]. Recurrent inguinal hernia was found in 94.9\% of men and in $5.1 \%$ of women, indicating that there were more men with a recurrent hernia than those with a primary hernia. Our data show that recurrent inguinal hernia was found in $5.5 \%$ of hernia repair operations. The proportion of recurrent inguinal hernia in our study sample was higher than the nationwide proportion as local clinics refer their patients with recurrent inguinal hernia to our institute for assessment and treatment.

This study provided definite data on the surgical problem of hernia recurrence. The maximum number of patients presenting with recurrent hernias were middle-aged men [13]. In this study, the incidence rate of right recurrent inguinal hernia was higher than that on the left side because the incidence inguinal hernia was higher on the right side than that on the left. A recent meta-analysis shows that direct inguinal hernia was a significant risk factor for recurrence after inguinal hernia surgery [14]. Our data showed that among patients with recurrent hernia, the ratio of patients with direct and indirect hernia was nearly the same (49.4\% vs. $47.1 \%$, respectively).

The high prevalence of early recurrence of hernia was predominantly due to technical failures, as opposed to late recurrences that were caused essentially by local tissue failure $[13,15,16]$. The recurrence rate of hernias was the highest within 1 year of surgery and the recurrence rate between 1 year and 2 years was the second highest. Moreover, we found that early recurrence was more likely in the mesh repair group compared 
to the tissue repair group (Table 4). Early recurrence was noted if a technical problem occurred during mesh repair, while late recurrence was observed in patients who underwent tissue repair [6].

Laparoscopic repair was recommended for he patients who underwent open primary inguinal hernia surgery and experienced recurrence [17]. However, in this study, surgeons tend to perform open surgery for patients who underwent open primary inguinal hernia surgery. In fact, in $28 \%$ of cases, surgeons did not follow the recommendation for laparoscopic surgery for recurrent hernia.

The present study has some limitations. First, our data set is insufficient to draw conclusion regarding the causation of recurrent hernia, and the 4 participating institutions may not be representative of all centers treating hernias in Korea. Second, we could not adjust for different operation methods or other unknown factors. However, there have been no recent reports on hernia recurrence in Asia and this large-scale multiinstitutional study shows the epidemiological characteristics of recurrent inguinal hernia in Korea.

From our results, we found that the rate of recurrent hernia was highest in the first year following hernia repair, was higher in men. Further research is needed to identify the causes of early recurrence of hernias and prevent their occurrence. The higher proportion of early recurrences following hernia repair may be explained by the high proportion of technical failures [6]

\section{CONFLICTS OF INTEREST}

No potential conflict of interest relevant to this article was reported.

\section{REFERENCES}

1. EU Hernia Trialists Collaboration. Repair of groin hernia with synthetic mesh: meta-analysis of randomized controlled trials. Ann Surg 2002;235:322-32.

2. Bendavid R. Inguinal herniorrhaphy in women. Hernia 2006:10:103-4.

3. EU Hernia Trialists Collaboration. Mesh compared with non-mesh methods of open groin hernia repair: systematic review of randomized controlled trials. $\mathrm{Br} \mathrm{J}$ Surg 2000;87:854-9.

4. Scott NW, McCormack K, Graham P, Go PM, Ross SJ, Grant AM. Open mesh versus non-mesh for repair of femoral and inguinal hernia. Cochrane Database Syst Rev 2002;(4):CD002197.

5. Dahlstrand U, Wollert S, Nordin P, Sandblom G, Gunnarsson U. Emergency femoral hernia repair: a study based on a national register. Ann Surg 2009;249:672-6.

6. Magnusson N, Nordin P, Hedberg M, Gunnarsson U, Sandblom G. The time profile of groin hernia recurrences. Hernia 2010; 14:341-4.

7. Bay-Nielsen M, Nordin P, Nilsson E, Kehlet H; Danish Hernia Data Base and the Swedish Hernia Data Base. Operative findings in recurrent hernia after a Lichtenstein procedure. Am J Surg 2001;182: 134-6.

8. Sondenaa K, Nesvik I, Breivik K, Korner H. Long-term follow-up of 1059 consecutive primary and recurrent inguinal hernias in a teaching hospital. Eur J Surg 2001;167: 125-9.

9. Matthews RD, Anthony T, Kim LT, Wang J, Fitzgibbons RJ Jr, Giobbie-Hurder A, et al. Factors associated with postoperative complications and hernia recurrence for patients undergoing inguinal hernia repair: a report from the VA Cooperative Hernia Study Group. Am J Surg 2007;194: 611-7.

10. Heller CA, Marucci DD, Dunn T, Barr EM, Houang M, Dos Remedios C. Inguinal canal "lipoma". Clin Anat 2002;15:280-5.

11. Eklund AS, Montgomery AK, Rasmussen IC, Sandbue RP, Bergkvist LA, Rudberg CR. Low recurrence rate after laparoscopic (TEP) and open (Lichtenstein) inguinal hernia repair: a randomized, multicenter trial with 5-year follow-up. Ann Surg 2009:249:33-8.

12. Han SR, Kim HJ, Kim NH, Shin S, Yoo RN,
Kim G, et al. Inguinal hernia surgery in Korea. Ann Surg Treat Res 2019;97:41-7.

13. Jansen PL, Klinge U, Jansen M, Junge K. Risk factors for early recurrence after inguinal hernia repair. BMC Surg 2009;9: 18.

14. Burcharth J, Pommergaard HC, Bisgaard T, Rosenberg J. Patient-related risk factors for recurrence after inguinal hernia repair: a systematic review and meta-analysis of observational studies. Surg Innov 2015;22: 303-17.

15. Phillips EH, Rosenthal R, Fallas M, Carroll B, Arregui M, Corbitt J, et al. Reasons for early recurrence following laparoscopic hernioplasty. Surg Endosc 1995;9:140-4.

16. Lowham AS, Filipi CJ, Fitzgibbons RJ Jr, Stoppa R, Wantz GE, Felix EL, et al. Mechanisms of hernia recurrence after preperitoneal mesh repair. Traditional and laparoscopic. Ann Surg 1997;225:42231.

17. HerniaSurge Group. International guidelines for groin hernia management. Hernia 2018;22:1-165. 